\title{
Area Prior Constrained Level Set Evolution for Medical Image Segmentation
}

\author{
Ismail Ben Ayed ${ }^{a}$, Shuo $\mathrm{Li}^{a}$, Ali Islam ${ }^{b}$, Greg Garvin ${ }^{b}$, and Rethy Chhem ${ }^{c}$ \\ ${ }^{a}$ GE Healthcare, London, Ontario, Canada; \\ ${ }^{b}$ St. Joseph's Health Care, London, Ontario, Canada; \\ ${ }^{c}$ London Health Sciences Center, London, Ontario, Canada
}

\begin{abstract}
The level set framework has proven well suited to medical image segmentation ${ }^{1-6}$ thanks to its ability of balancing the contribution of image data and prior knowledge in a principled, flexible and transparent way. It consists of evolving a curve toward the target object boundaries. The curve evolution equation is sought following the optimization of a cost functional containing two types of terms: data terms, which measure the fidelity of segmentation to image intensities, and prior terms, which traduce learned prior knowledge. Without priors many algorithms are likely to fail due to high noise, low contrast and data incompleteness. Different priors have been investigated such as shape ${ }^{1}$ and appearance priors. ${ }^{7}$ In this study, we propose a simple type of priors: the area prior. This prior embeds knowledge of an approximate object area and has two positive effects. First, It speeds up significantly the evolution when the curve is far from the target object boundaries. Second, it slows down the evolution when the curve is close to the target. Consequently, it reinforces curve stability at the desired boundaries when dealing with low contrast intensity edges. The algorithm is validated with several experiments using Magnetic Resonance (MR) images and Computed Tomography (CT) images. A comparison with another level set method illustrates the positive effects of the area prior.
\end{abstract}

Keywords: Image segmentation, level set, curve evolution, area prior, MRI, CT.

\section{INTRODUCTION}

Active contour/level set segmentation methods, which use an active curve to delineate the target object boundaries, have been generally effective. The curve evolution equations are derived from the optimization of a global objective functional. The level set representation of an active curve ${ }^{14}$ yields numerically stable and efficient algorithms. It handles automatically topological changes of the evolving curve and yields an implicit representation of the corresponding region membership and boundary, which removes the need of complex data structures. Several studies have shown that the variational, active contour/level set formalism can lead to effective algorithms to solve many vision problems such as tracking, ${ }^{16}$ motion estimation, ${ }^{17}$ and $3 \mathrm{D}$ interpretation, ${ }^{18}$ as well as many medical image analysis problems. ${ }^{1-3}$ This formalism is well suited to medical image segmentation because it can balance the contribution of image data and prior knowledge in a principled, flexible and transparent way. Level set methods are commonly based on the optimization of an objective functional which contains two types of terms: data terms, which measure the fidelity of segmentation to image intensities, and prior terms, which traduce prior knowledge learned from a set of relevant images and segmentation examples. Data terms can be divided into two categories: region-based ${ }^{8,10,11}$ and contour-based data terms. ${ }^{12,15}$ In this study, we use a contour-based data term in conjunction with a new simple prior: tha area prior. The latter embeds knowledge of an approximate object area (size). As we will show in the experiments (section 4), the area prior has two positive effects. First, It speeds up significantly the evolution when the curve is far from the target object boundaries. Second, it slows down the evolution when the curve is close to the target. This reinforces curve stability at the desired boundaries when dealing with low contrast intensity edges.

The remainder of this paper is organized as follows: In the next section, we give the necessary background to introduce the proposed method. In section 3, we define an area prior and derive the corresponding level set curve evolution equation. In section 4, we describe representative experiments and comparisons with Magnetic Resonance (MR) images and Computed Tomography (CT) images.

Further author information: (Send correspondence to Ismail Ben Ayed)

Ismail Ben Ayed: E-mail: ismail.benayed@ge.com, Telephone: 15199026822

Medical Imaging 2008: Image Processing, edited by Joseph M. Reinhardt, Josien P. W. Pluim,

Proc. of SPIE Vol. 6914, 691402, (2008)

1605-7422/08/\$18 - doi: 10.1117/12.770579

Proc. of SPIE Vol. 6914 691402-1 


\section{BACKGROUND}

Let $I: \Omega \subset R^{2} \rightarrow R$ be an image function. Consider a closed planar curve $\vec{\Gamma}(s):[0,1] \rightarrow \Omega$ parameterized by $s$. A contour-based data term is commonly expressed in terms of the image gradient ${ }^{12,15}$

$$
\mathcal{F}_{D}(\vec{\Gamma}, I)=\oint_{\vec{\Gamma}} g(I(s)) d s
$$

where function $g$ is generally given by

$$
g(I)=\frac{1}{1+\left|\nabla G_{\sigma} * I\right|^{p}}, p=1,2 .
$$

and $G_{\sigma}$ is the Gaussian kernel with standard deviation $\sigma$

$$
G_{\sigma}(x, y)=\frac{1}{\sqrt{\sigma}} e^{-\frac{\left|x^{2}+y^{2}\right|}{4 \sigma}},(x, y) \in \Omega
$$

The evolution equation of $\vec{\Gamma}$ is obtained by minimizing $\mathcal{F}_{D}(\vec{\Gamma}, I)$ with respect to $\vec{\Gamma}$. Function $g(\mathrm{I})$ attracts $\vec{\Gamma}$ toward high gradients of intensities, i.e., toward the boundaries of segmentation. In order to speed up the curve evolution, a weighted area term, $\mathcal{A}_{g}$, can be added to (1) as follows ${ }^{12}$

$$
\mathcal{F}_{D, \mathcal{A}_{g}}(\vec{\Gamma}, I)=\oint_{\vec{\Gamma}} g(I(s)) d s+\underbrace{}_{\mathcal{A}_{g}: \underbrace{\mu \int_{\mathbf{R}_{\vec{\Gamma}}^{C}} g(I(\mathbf{x})) d \mathbf{x}}_{\text {Weighted area term }}}
$$

Where $\mathbf{R}_{\vec{\Gamma}}^{C}$ denotes the region outside curve $\vec{\Gamma}$. Coefficient $\mu$ can be positive or negative, depending on the initial position of the curve. Suppose that the initial curve is inside the target object. In this case, a positive value of $\mu$ corresponds to decreasing the area outside $\vec{\Gamma}$, and consequently speeds up the evolution toward the desired boundaries. When the initial curve is outside the target object, $\mu$ should be negative.

Contour-based data terms have been widely used in general-purpose image segmentation ${ }^{12,15}$ and in many important applications such as in medical image analysis. ${ }^{1}$ However, relying on the sole contribution of the data term might be insufficient when dealing with low contrast (small intensity gradient), blurry edges, and high noise. In such cases, function $g(I)$ is weak and models (1) and (4) are likely to fail in stopping the curve at the sought boundaries. In section 4 , we will show an example where the curve evolution fails in segmenting the target object with model (4). Prior knowledge is essential to overcome high noise, low contrast and data incompleteness. Different priors have been investigated such as shape ${ }^{1,8}$ and appearance priors. ${ }^{7}$ In this study, we investigate a simple type of priors: the area priors. In the next section, we will define an area prior and briefly discuss the positive effects of such a prior.

\section{THE PROPOSED AREA PRIOR}

We propose to embed an area prior in functional (4) as follows

$$
\mathcal{F}_{D, \mathcal{A}_{g}, \mathcal{A}_{p}}(\vec{\Gamma}, I)=\oint_{\vec{\Gamma}} g(I(s)) d s+\underbrace{\frac{1}{\mathcal{A}_{p}^{2}}\left(\int_{\mathbf{R}_{\vec{\Gamma}}} d \mathbf{x}-\mathcal{A}_{p}\right)^{2}}_{\text {Area prior }} \underbrace{\mu \int_{\mathbf{R}_{\vec{\Gamma}}^{C}} g(I(\mathbf{x})) d \mathbf{x}}_{\mathcal{A}_{g}}
$$

where $\mathbf{R}_{\vec{\Gamma}}^{C}$ is the region inside curve $\vec{\Gamma}$, and $\mathcal{A}_{p}$ is an approximate prior value of the target object area (size). For a given class of images or for a specific application, $\mathcal{A}_{p}$ is determined over a set of relevant images and segmentation examples. We assume that for a given application, the area of the target object does not vary much from an image to another. This assumption is valid in many medical image segmentation problems because we have generally to deal with a particular organ or structure. For instance, the variation of the spine area over different MR images is small. To understand the effect of the area prior in (5), we assume without loss of generality that 
the initial curve is inside the target object ( $\mu$ should be positive). In this case, the outward motion of $\vec{\Gamma}$ decreases both the area prior and the weighted area $\mathcal{A}_{g}$. This speeds up significantly the evolution when the curve is far from the target object boundaries. When curve $\vec{\Gamma}$ is close to desired boundaries, the area prior approaches zero and the second term in (5) (the product of the area prior and $\mathcal{A}_{g}$ ) becomes close to its global minimum (zero). This slows down significantly the evolution to stabilize the curve at the desired boundaries. In the experiment section, we will illustrate those positive effects of the area prior by comparisons with model (4). ${ }^{12}$

\subsection{Level set representation of $\vec{\Gamma}$}

A level set implementation ${ }^{14}$ of curve evolution consists of representing curve $\vec{\Gamma}$ implicitly by the zero level set of a function $u: \Omega \subset R^{2} \rightarrow R$, i.e., $\vec{\Gamma}$ is the set $\{u=0\}$. The region inside $\vec{\Gamma}$ corresponds to $u<0$, and the region outside $\vec{\Gamma}$ corresponds to $u>0$. The level-set representation has well-known advantages over an explicit discretization of $\vec{\Gamma}$ using a number of points on the curve. It handles automatically topological changes of the evolving curve $-\vec{\Gamma}$ may split and merge while $u$ remains a function-and can be effected by stable numerical schemes. ${ }^{14}$ Using the level set representation in functional (5), we obtain

$$
\mathcal{F}_{D, \mathcal{A}_{g}, \mathcal{A}_{p}}(u, I)=\int_{\Omega} g(I) \delta(u)|\nabla u| d \mathbf{x}+\underbrace{\frac{1}{\mathcal{A}_{p}^{2}}\left(\int_{\Omega} H(u) d \mathbf{x}-\mathcal{A}_{p}\right)^{2}}_{\text {Area prior }} \underbrace{\mu \int_{\Omega} g(I) H(-u) d \mathbf{x}}_{\mathcal{A}_{g}}
$$

where $H$ is the Heaviside function and $\delta$ is the Dirac function.

\subsection{The obtained level set evolution equation}

The level set evolution equation minimizing $\mathcal{F}_{D, \mathcal{A}_{g}, \mathcal{A}_{p}}(u, I)$ is obtained by embedding $u$ in a one-parameter family of functions: $u(\mathbf{x}, t): \Omega \times R^{+} \rightarrow R$, and solving the partial differential equation (PDE)

$$
\frac{\partial u(\mathbf{x}, t)}{\partial t}=-\frac{\partial \mathcal{F}_{D, \mathcal{A}_{g}, \mathcal{A}_{p}}(u, I)}{\partial u}
$$

The functional derivative of $\mathcal{F}_{D, \mathcal{A}_{g}, \mathcal{A}_{p}}(u, I)$ with respect to $u$ is obtained using the standard calculus of the Euler-Lagrange equation. This leads to the following evolution equation

$$
\begin{aligned}
\frac{\partial u(\mathbf{x}, t)}{\partial t} & =\delta(u) \operatorname{div}\left(g(I) \frac{\nabla u}{|\nabla u|}\right)+\mu g(I) \delta(u) \frac{1}{\mathcal{A}_{p}^{2}}\left(\int_{\Omega} H(u) d \mathbf{x}-\mathcal{A}_{p}\right)^{2} \\
& -\mu \int_{\Omega} g(I) H(-u) d \mathbf{x} \frac{2}{\mathcal{A}_{p}^{2}}\left(\int_{\Omega} H(u) d \mathbf{x}-\mathcal{A}_{p}\right) \delta(u)
\end{aligned}
$$

In practice, the Heaviside and Dirac functions in the evolution equation are commonly smoothed according to ${ }^{9}$

$$
\begin{gathered}
H_{\epsilon}(z)=\left\{\begin{array}{l}
1 \text { if } z>\epsilon \\
0 \text { if } z<-\epsilon \\
\frac{1}{2}\left[1+\frac{z}{\epsilon}+\frac{1}{\pi} \sin \left(\frac{\pi z}{\epsilon}\right)\right] \text { if }|z| \leq \epsilon
\end{array}\right. \\
\delta_{\epsilon}(z)=H_{\epsilon}^{\prime}(z)
\end{gathered}
$$

where $\epsilon>0$.

\section{EXPERIMENTS AND COMPARISONS}

We applied the method successfully to several MR images and CT images. We first show a representative result of detecting vertebral bodies in MR images of the spine. This task is still challenging due to the poor contrast between the bones and the surrounding tissue. Very few investigations have addressed this task. ${ }^{13}$ To illustrate clearly the positive effect of the area prior, we show an example where our method succeeds in detecting the 
vertebral bodies in a spine image while the method in ${ }^{12}$ fails. We show in Figure 1 results with our method, i.e., with the area prior (first line), and with the method in, ${ }^{12}$ i.e., without the area prior (second line). The same initialization (Figure $1 \mathrm{a}$ ) and parameters are used for both algorithms. The approximate object size is estimated over several spine images. At iteration 40, the curve is very close to the final position with our method (Figure $1 \mathrm{~b}$ ) while it is still close to the initialization with the method in ${ }^{12}$ (Figure $1 \mathrm{e}$ ). This difference between the two methods illustrates, on one hand, how the area prior makes the evolution faster when the curve is far from the desired boundaries. On the other hand, the area prior stabilizes the curve near the desired boundaries as shown in Figure $1 \mathrm{c}$ (iteration 300) and Figure $1 \mathrm{~d}$ (iteration 500). Figures $1 \mathrm{f}$ and $\mathrm{g}$ depict how the curve passes through the desired boundaries without the area prior (method in ${ }^{12}$ ). The use of the area prior permits to detect accurately the vertebral boundaries.

We also show in Figure 2 representative examples of detecting the spleen in two CT images. The area prior permits to detect accurately the spleen in both images as shown in Figures $2 \mathrm{c}$ and d, while the method in ${ }^{12}$ fails as shown in Figure $2 \mathrm{~g}$ and $\mathrm{h}$. The area prior speeds up the evolution as depicted in $\mathrm{b}$ (with the area prior) and $\mathrm{f}$ (without the area prior).

\section{CONCLUSION}

We proposed an area prior for level set curve evolution segmentation. This prior embeds knowledge of an approximate object area and has two positive effects. First, It speeds up significantly the evolution when the curve is far from the target object boundaries. Second, it slows down the evolution when the curve is close to the target. Results and comparisons illustrate the positive effects of the area prior. For many object detection problems in medical imaging, the area prior may be a sufficient, simple, and fast alternative to cumbersome, computationally expensive priors such as shape priors.

\section{REFERENCES}

1. N. Paragios, "A level set approach for shape-driven segmentation and tracking of the left ventricle," IEEE Transactions on Medical Imaging 22(6), pp. 773-776, 2003.

2. Shuo Li, Thomas Fevens, Adam Krzyzak, Chao Jin, and Song Li, "Semi-Automatic Decay Detection on Dental X-rays using Variational Level Set," Pattern Recognition 40(10), pp. 2861-2873, 2007.

3. Shuo Li, Thomas Fevens, Adam Krzyzak and Song Li, "Fast and Robust Clinical Triple-region Image Segmentation using One Level Set Function," MICCAI, LNCS, pp. 766-773, 2006.

4. G. Slabaugh, G. Unal, T. Fang, and M. Wels, "Ultrasound-Specific Segmentation via Decorrelation and Statistical Region-Based Active Contours," Proc. CVPR, pp. 45-53, 2006.

5. M. Holtzman-Gazit, R. Kimmel, N. Peled, and D. Goldsher, "Segmentation of thin structures in volumetric medical images," IEEE Transactions on Image Processing, 15(2), pp. 354-363, 2006.

6. C. S. Drapaca, V. Cardenas, and C. Studholme, "Segmentation of tissue boundary evolution from brain MR image sequences using multi-phase level sets," Computer Vision and Image Understanding, 100(3), pp. 312-329, 2005.

7. T. Zhang and D. Freedman, "Improving performance of distribution tracking through background mismatch," IEEE Transactions on Pattern Analysis and Machine Intelligence, 27(2), pp. 282-287, 2005.

8. D. Cremers, M. Rousson and R. Deriche, "A review of statistical approaches to level set segmentation: integrating color, texture, motion and shape," International Journal of Computer Vision 72(2), pp. 195-215 , 2007.

9. T. F. Chan and L. A. Vese, "Active Contours without Edges," IEEE Transactions on Image Processing 10(2), pp. 266-277, 2001.

10. Ismail Ben Ayed, N. Hennane, and A. Mitiche, "Unsupervised Variational Image Segmentation/Classification using a Weibull Observation Model," IEEE Transactions on Image Processing 15(11), pp. 3431-3439, 2006.

11. Ismail Ben Ayed, Amar Mitiche, and Ziad Belhadj, "Polarimetric Image Segmentation via MaximumLikelihood Approximation and Efficient Multiphase Level-Sets," IEEE Transactions on Pattern Analysis and Machine Intelligence 28(9), pp. 1493-1500, 2006. 


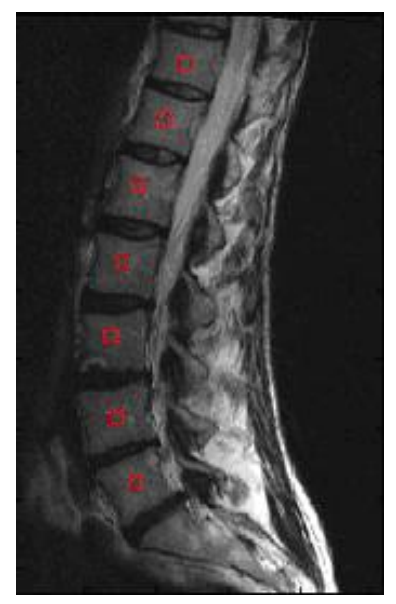

(a) Initialization

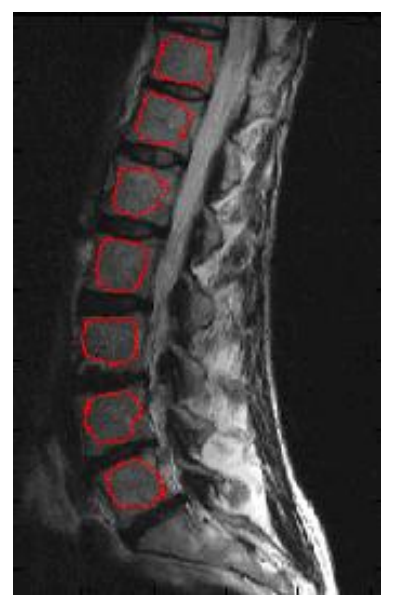

(b) iteration 40

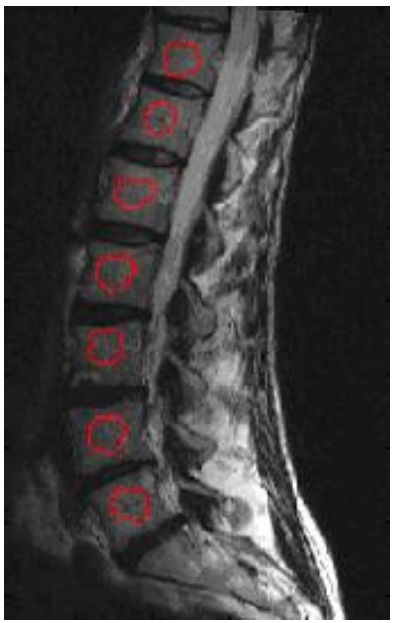

(e) iteration 40

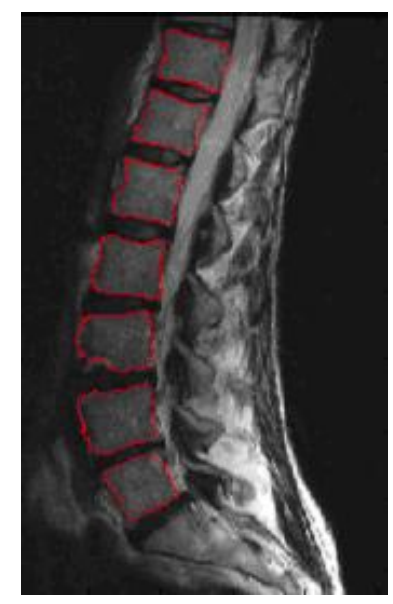

(c) iteration 300

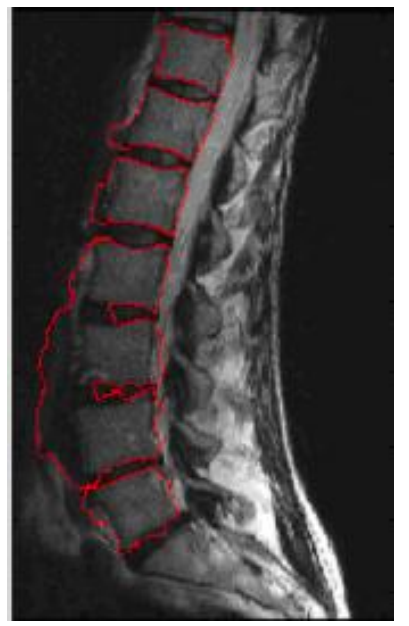

(f) iteration 300

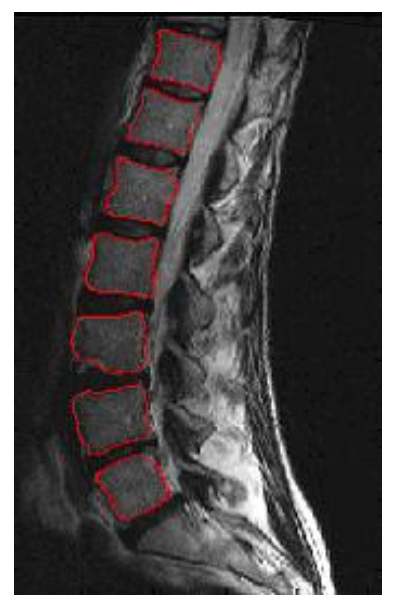

(d) iteration 500

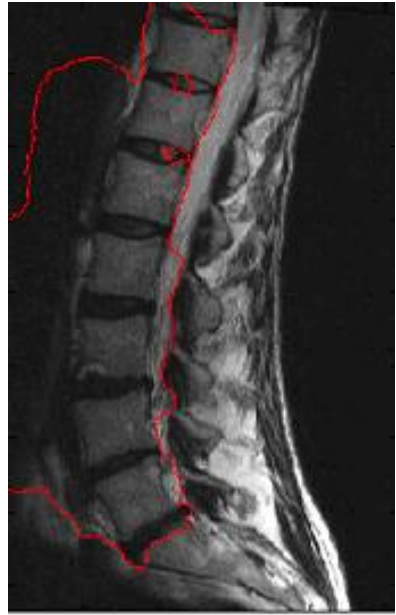

iteration 500

Figure 1. Curve evolution results with our method, i.e., with the area prior (first line), and with the method in, ${ }^{12}$ i.e., without the area prior (second line). (a): curve initialization for both methods. First line: (b), (c), and (d) depict the position of the curve with the area prior respectively at iterations 40, 300, and 500. Second line: (e), (f), and (g) depict the position of the curve with the method in ${ }^{12}$ at the same iterations. $\mathcal{A}_{p}=11.10^{3} . \mu=5$.

12. C. Li, C. Xu, C. Gui, and M. D. Fox, "Level Set Evolution without Re-Initialization: A New Variational Formulation," IEEE CVPR 1, pp. 430-436, 2005.

13. J. Carballido-Gamio, S. J. Belongie, and S. Majumdar, "Normalized Cuts in 3-D for Spinal MRI Segmentation," IEEE Transactions on Medical Imaging 23(1), pp. 36-44, 2004.

14. J. A. Sethian, "Level set methods and fast marching methods," Cambridge University Press, 1999.

15. V. Caselles, R. Kimmel, and G. Sapiro, "Geodesic active contours," International Journal of Computer Vision 22, pp. 61-79, 1997.

16. D. Cremers, "Dynamical statistical shape priors for level set based tracking," IEEE Transactions on Pattern Analysis and Machine Intelligence, 28(8), pp. 1262-1273, 2006.

17. C. Vazquez, A. Mitiche, and R. Laganiere, "Joint Multiregion Segmentation and Parametric Estimation of 


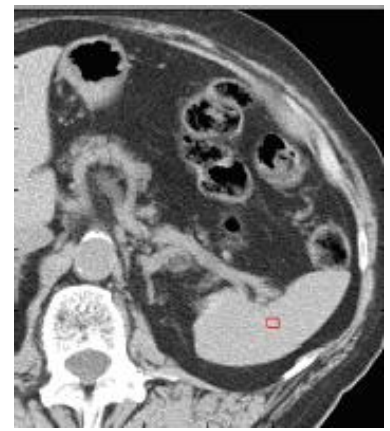

(a) Initial curve (Image 1)

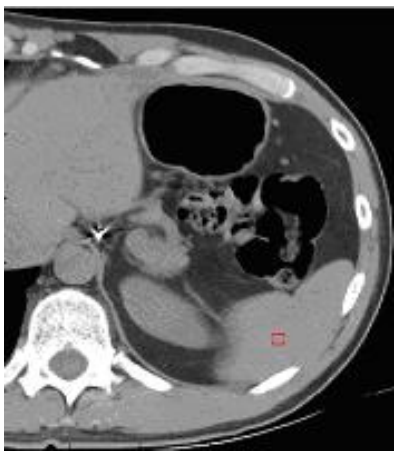

(e) Initial curve (Image 2)

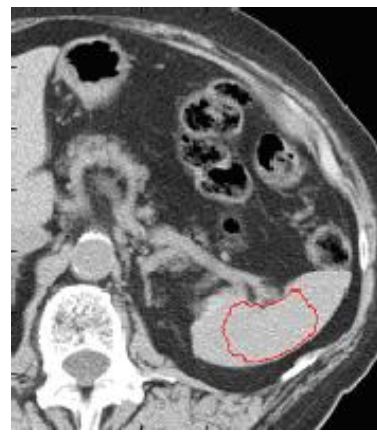

(b) iteration 40

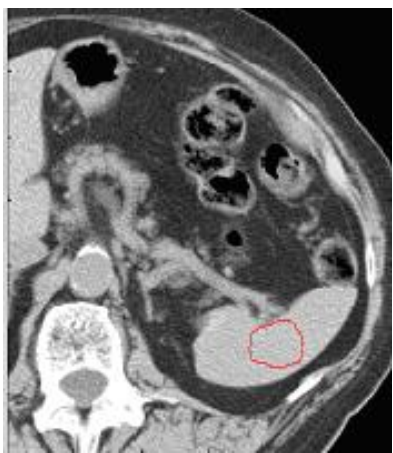

(f) iteration 40

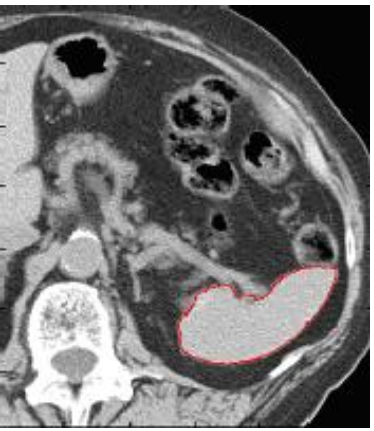

(c) iteration 400 (convergence)

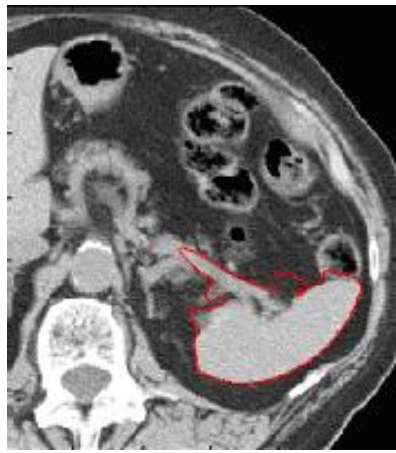

(g) iteration 400

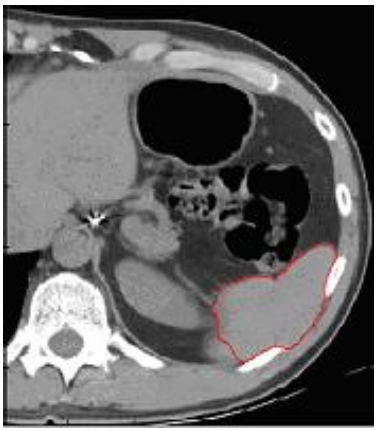

(d)

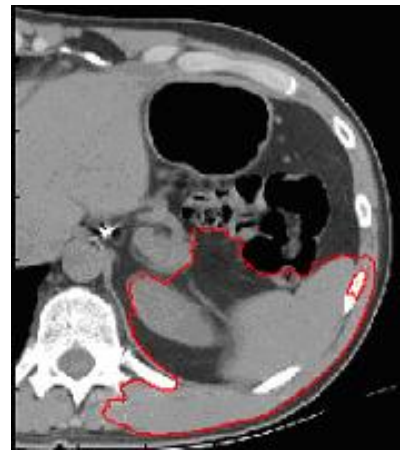

(h)

Figure 2. Curve evolution results for spleen detection in two CT images with our method, i.e., with the area prior (first line), and with the method in, ${ }^{12}$ i.e., without the area prior (second line). (a), (e): the two images with the initial curve. First line: (b) and (c) depict the position of the curve with the area prior respectively at iterations 40,400 (first image). Second line: (f) and (g) depict the position of the curve with the method in ${ }^{12}$ at the same iterations. (d) and (h) show the results (second image) respectively with our method and with the method in. ${ }^{12}$

Image Motion by Basis Function Representation and Level Set Evolution," IEEE Transactions on Pattern Analysis and Machine Intelligence, 28, pp. 782-793, 2006.

18. A. Mitiche and H. Sekkati, "Optical Flow 3D Segmentation and Interpretation: A Variational Method with Active Curve Evolution and Level Sets," IEEE Transactions on Pattern Analysis and Machine Intelligence, 28(11), pp. 1818-1829, 2006. 